\title{
Baetis numidicus n.sp., Ephéméroptère nouveau d'Algérie (Baetidae)
}

\author{
T. Soldán 1 \\ A.G.B. Thomas ${ }^{2}$
}

Description illustrée de la larve au dernier stade et de l'imago ơ de Baetis numidicus $\mathbf{n}$. sp. Cette espèce, rencontrée dans le Nord de l'Algérie, présente à l'état larvaire des affinités morphologiques avec $B$ gracilis Bogoescu et Tabacaru.

Baetis numidicus n. sp., a new mayfly from Algeria (Ephemeroptera, Baetidae).

The nymph (last instar) and the $\sigma$ imagine of $B$. mumidicus $n$. sp. from northern Algeria are described and illustrated. The nymphal stage of this species is related to that of $B$. gracilis Bogoescu and Tabacaru.

L'étude par l'un de nous (T.S.) de la faune benthique du réseau hydrographique de l'oued Chiffa, près d'Alger, a permis la découverte d'une espèce nouvelle du genre Baetis. La correspondance entre larve et adulte a été établie par élevage.

\section{t. - Larve au dernier stade.}

Diagnuse sommaire : espèce de petite taille, brun foncé assez uni - a l'exception des tergites 8 et 9 très clairs -, et à paracerque long ( $2 / 3$ de la longueur des cerques environ).

TÉTE.

Presque entièrement brun foncé, un peu plus grisâtre dans la région antéro-inférieure. Traces d'in. sertions musculaires du crâne peu marquées (larve 8).

Antennes : en plus de quelques soies, le pédicelle porte des impressions concaves (fig. 1).

Le labre (fig. 2), à l'apex arrondi et fortement incisé, porte $1+2$ soies frontales principales et la trace d'insertion de plusieurs autres soies plus petites. Il est relativement proche de celui de $B$. graci-

1. Institute of Entomology. Czechoslovak Acaderny of Sciences, Na Sadkach 702. CS 37005 Ceské Budejovice, Crechuslovakia.

2. Laboratoire d'Hydrobiologie, ERA 702 du C.N.R.S., U.P.S., 118 route de Narbonne, F 31062 Toulouse Cedex, France. lis Bogoescu (selon la figure 140 a de MüllerLiebenau, 1969) et présente un rapport largeur/longueur - soies bordantes exclues - de 1,35 sur l'holotype. Les soies marginales (antérieures et laterales) sont tottes ramifiées.

Maxilles : palpe maxillaire à apex tronconique (fig. 3) porteur d'une dent peu visible et de petites soies assez nombreuses.

Mandibules (fig. 4) à région incisive nettement den. telée et prosthecas droite et gauche très asymétri. ques mais aucune forte soie près de la prostheca droite, contrairement à $B$. gracilis selon Müller. Liebenau (t.c., fig. 140b).

Labium : ensemble des $2^{e}$ et $3^{e}$ articles du palpe (fig. 5) présentant sur l'holotype un rapport longueur/largeur maximum voisin de 2,25 et un contour externe régulièrement arrondi; $3^{\mathbf{e}}$ article légèrement saillant du côté interne. Paraglosses (fig. 6) assez étroites et fortement incurvées avec peu de soies apicales, disposées sur deux rangs seulement. Glosses: fig. 7.

\section{THORAX}

Coloration assez uniforme, peu contrastée, brun foncé sáuf ventralement oủ elle est blanchâtre. Pat. tes claires, blanchâtres, à l'exception d'une large bande brun foncé cousrant la moitie distale du fémur : cette bande renfermé une tache claire subapicale, vers le bord posterieur. 

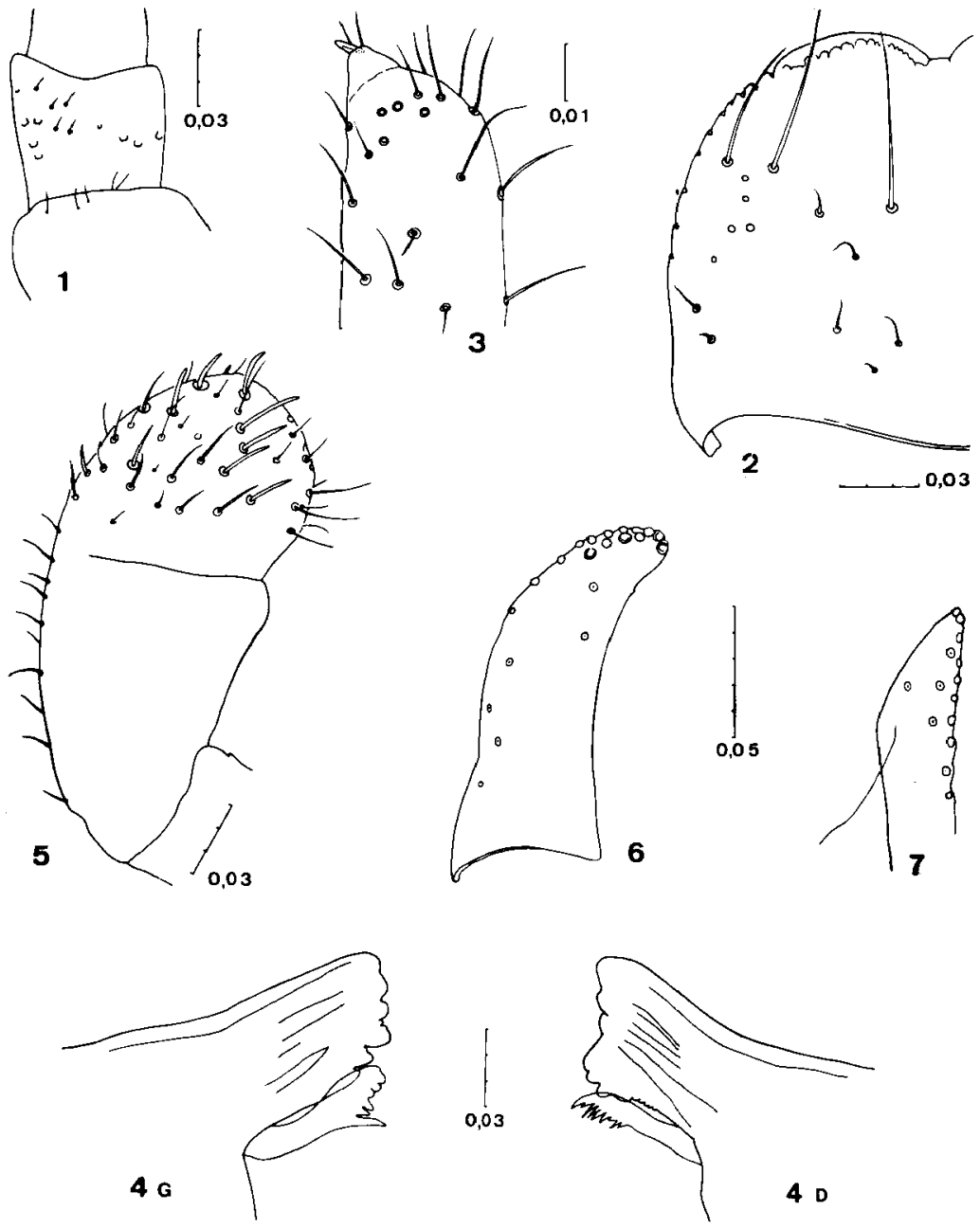

Fig. 1 à 7.- Larve $Q$ de Baetis numidicus n. sp. au dernier stade. Echelle en $\mathrm{mm}$.

1 : pédicelle. 2 : labre. 3 : apex du palpe maxillaire. 4 :

apex et prostheca des mandibules droite (di et gauche (g) $5: 2^{e}$ et $3^{\mathrm{e}}$ articles du palpe labial, face ventrale. 6 : paraglosse et 7 : glosse, face ventrale. 


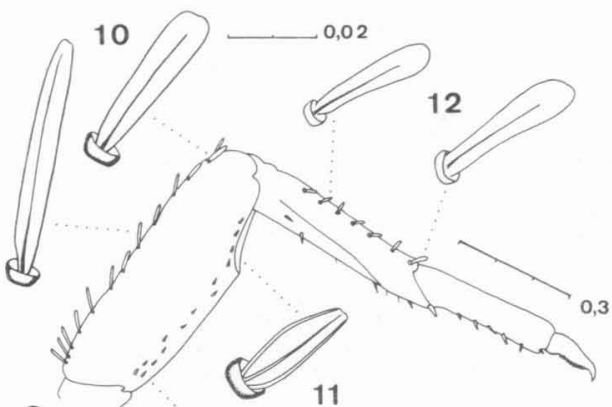

8

11
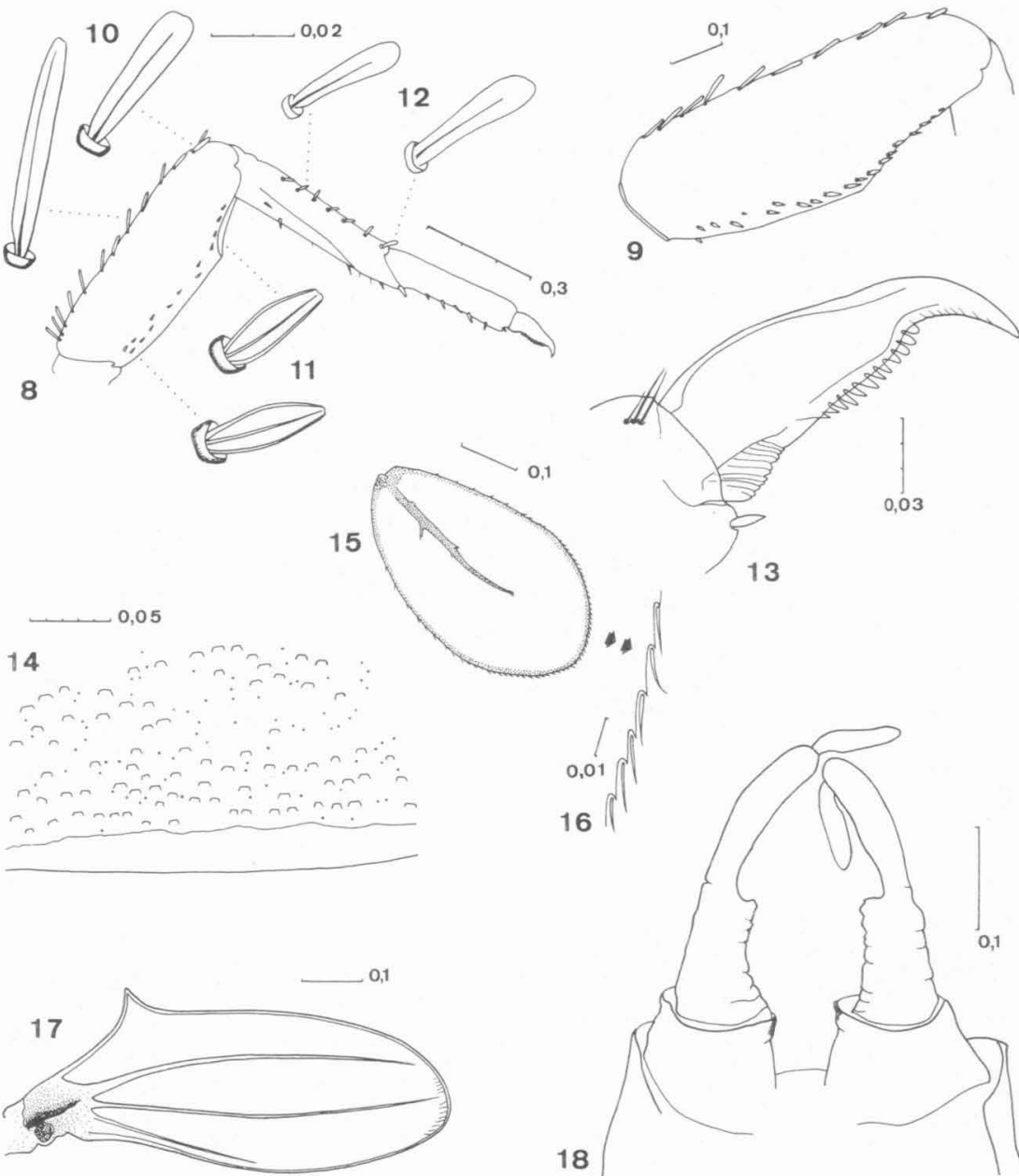

Fic. 8 à 16. - Larve $\odot$ de Baetis numidicus n. sp. au dernier stade. Echelle en $\mathrm{mm}$.

8: vue d'ensemble dorsale de la patte II. 9 : vue dorsale du fémur $\mathrm{I}$. 10 et 11 : écailles du fémur, bords postéroexterne (10) et antéro-interne (11). 12 : écailles externes du tibia. 13 : griffe tarsale. 14 : ornementation cuticu.

laire et bord postérieur du 5e tergite abdominal. $15: 5$ e branchie et 16 : son ornementation marginale.

Fig. 17 et 18- Imago $\sigma$ de Baetis numidicus n. sp. Echelle en mm.

17: aile postérieure. 18: styligère et styles en vue ventrale. 
Les pattes sont massives (fig. 8). Fémurs très larges - surtout le $1^{\text {er }}$ (fig. 9) - avec une forte concavité à l'apex, du côté interne: une douzaine de longues écailles arrondies (fig. 10), non dentelées et entremêlées de soies très fines, sur le bord postéroexterne ; sur la surface dorsale, des impressions à concavité dirigée vers l'extrémité distale ; de fortes écailles, épaisses et aiguës (fig. 11) sur le bord antérointerne, en particulier le long de la concavite préarticulaire apicale. Tibias épais, sensiblement plus courts que les fémurs (rapport des longueurs tibia/fémur + tarse voisin de 0,5 ), portant de longues écailles non dentelées, élargies êt arrondies à l'extrémité (fig. 12). Griffes tarsales (fig. 13) larges à la base et brusquement rétrécies et recourbées assez près de l'apex, avec $12-13$ petites denticulations subégales.

\section{ABDOMEN.}

Dorsalement brun foncé uni, à l'exception, sur chaque tergite, de deux taches linéaires obliques, symétriques, fines et claires, peu visibles qui précèdent deux petits points blanchâtres, eux aussi peu apparents. Le $9 c$ tergite est entièrement blanchâtre et le $8^{e}$ presque entièrement, hormis deux bandes latérales brunes recouvertes par la dernière paire de branchies. Ventralement. l'abdomen est brun grisàtre clair avec deux séries de maculations latérales brunes, longitudinales et limitées à la moitié antérieure des sternites. $9^{e}$ sternite entièrement clair.

La surface des tergites (fig. 14) porte des impressions concaves assez anguleuses mais, caractère très remarquable, le bord postérieur ne porte aucune denticulation.

Branchies ovoìdes, presque symétriques (fig. 15) et plutót longues (la plupart comme un tergite et demi) avec un tronc trachéen longitudinal violacé, bien visible. Bord épaissi et portant de nombreuses petites denticulations et soies, surtout dans la partie apicale (fig. 16).

Cerques brunâtre clair.

TAILLE (holotype : larve ९).

Longueur du corps : $4,0 \mathrm{~mm}$; des cerques : 2,5 $\mathrm{mm}$; du paracerque : $1,7 \mathrm{~mm}$.

\section{2. - Imago $\sigma$.}

Longueur du corps : 3,5 à $3,8 \mathrm{~mm}$, de l'aile antérieure: 5,2 à $5,6 \mathrm{~mm}$.
Tète et thorax sombres, brun noirâtre uniforme ; antennes à funicule plus clair. Yeux en turban brun rougeàtre, face supérieure à ommatidies légèrement plus foncée, paroi latérale avec un large anneau noir brunâtre. Ces yeux sont ovales en vue dorsale, à peu près deux fois plus longs que larges. Segments abdominaux I et VII à $X$ brun clair uniforme, tergites II à V'I brun jaunâtre clair, translucide. Stigmates abdominaux cerclés de noir intense. Pattes antérieures brun jaunâtre, tarse plus court d'un tiers que le tibia; pattes moyennes et postérieures plus claires.

Ailes antérieures transparentes légèrement brunâtres, à nervures longitudinales brun jaunâtre. Ailes postérieures ( $/ \mathrm{ig}$. 17) 2,5 fois plus longues que larges, avec trois nervures longitudinales simples, non bifurquées ; processus costal marqué et aigu.

Pince génitale blanchâtre sauf le segment basal

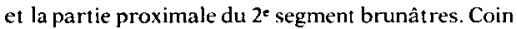
postéro-interne du segment basal (fig. 18) assez anguleux et nettement saillant; segment apical très long (aussi long que le quart du $2^{e}$ segment) et recourbé à la base. Cerques uniformément blanchâtres.

Imago $q$, subimagos of $\mathrm{e} \ell$ inconnues.

\section{3. - Matériel examiné.}

1 larve $९$ au dernier stade (holotype) et 3 imagos $\sigma$ (paratypes) obtenus par élevage, provenant de l'Oued Chiffa dans les gorges de la Chiffa (Wilaya de Blida, environs d'Alger) vers $200 \mathrm{~m}$ d'altitude. Captures du 6 au 9-X-1981 (T. Soldán leg.). L'holotype et deux paratypes sont déposés à l'Institut d'Entomologie de Ceské Budejovice, le troisième paratype au laboratoire d'Hydrobiologie de Toulouse. Conservation en alcool à $70^{\circ}$.

\section{4. - Affinités systématiques.}

B. numidicus n.sp. présente à l'état larvaire plusieurs caractères qui l'apparentent à $B$. gracilis Bogoescu et Tabacaru, 1957. Ces caractères touchent essentiellement la morphologie générale des pièces buccales et du corps. Cependant, B. numidicus diffère sensiblement de $B$. gracilis par:

- la coloration foncée uniforme des tergites 1 à 7 :

- l'absence de fortes soies près de la prostheca droite :

- l'absence de toute denticulation sur le bord postérieur des tergites;

- les tibias plus courts, comme chez B. niger. 
Les adultes de $B$. numidicus ne sont étroitement apparentés à aucun des groupes d'espèces niger, gracilis ou muticus. Ils peuvent en être distingues par leur aile postérieure relativement longue, dépourvue de bifurcation sur la seconde nervure et par l'absence de nervures intercalaires. La couleur des yeux rapproche $B$. numidicus de $B$. gracilis mais la morphologie des forceps est plus voisine de $B$. niger et de $B$. digitatus (segment apical très long).

\section{Travaux cites}

Muler Llebenau (I.). 1969.- Revision der europäischen Arten der Gattung Baelis Leach, 1815 (Insecta, Ephemeroptera). Gewäss A hu:äss. 48-49:1-214

M(tu.er-Liebenau (L.). 1974. - Baetidae aus Südfrankreich, Spanien und Porlugal (Insecta. Ephemeroplera). Gewäss. Abwäss. $53.54 \div 7-42$

Soldan (T.j et THomas (A.G.B.). 1983.- New and little-know species of mayflies (Ephemeroptera) from Algeria. Acta ent. bohemos lov. $80(5): 356.376$ 